\title{
Antecedentes maternos prenatales y riesgo de complicaciones neonatales en productos de término de bajo peso para edad gestacional
}

\author{
Karina L. Arce-López'1, Juan Vicencio-Rivas², José Iglesias-Leboreiro ${ }^{1,2}$, Isabel Bernárdez-Zapata ${ }^{1,2}$, \\ Mario E. Rendón-Macías ${ }^{2 \star}$ y Ariela Braverman-Bronstein ${ }^{2}$ \\ ${ }^{1}$ Facultad Mexicana de Medicina, Universidad La Salle; ${ }^{2}$ Hospital Español de México. Ciudad de México, México
}

\begin{abstract}
Resumen
Introducción: El bajo peso neonatal (<2.5 kg o < percentil 10) se atribuye a causas constitucionales (pequeño para la edad gestacional [PEG]) o a la restricción en el crecimiento intrauterino (RCIU), con riesgos posnatales diferentes. En ausencia de una valoración fetal-placentaria prenatal adecuada, resulta difícil establecerlo. El conocer los antecedentes maternos de enfermedades gestacionales (AMEG): hipertensión arterial (HTA), hipotiroidismo, Diabetes Mellitus (DM) gestacional y otra; pudiera orientar a la diferenciación y el manejo. El objetivo de este trabajo fue determinar si la presencia de AMEG se asocia a complicaciones neonatales en productos de bajo peso neonatal. Métodos: Se realizó un estudio de cohorte retrospectivo en un grupo de 349 recién nacidos vivos de término ( $\geq 37$ semanas de edad gestacional [SEG]), PEG, en cunero fisiológico y agrupados según los AMEG. Se determinó la frecuencia de dificultad respiratoria (DR), hipoglucemia (HG) e hiperbilirrubinemia (HBr). Resultados: El 16.6\% (58/349) presentó AMEG (58.6\% HTA y 41.3\% hipotiroidismo, solos o combinados). Los neonatos con AMEG fueron más limítrofes (37 SEG, $55.2 \%$ vs. 35.1\%; $p=0.037$ ). Los de 37 SEG con menor peso (diferencia de $\approx 100 \mathrm{~g}$; $p=0.028$ ), más riesgo de $H G$ (13.6\%; intervalo de confianza al 95\% [IC 95\%]: -4.08-31.2) pero menos DR (diferencia de $-4.7 \%$; IC 95\%: -20.6-11.05). La HG en los de 39 SEG solo se presentó en neonatos sin AMEG (diferencia 12.7\%; IC 95\%: 3.9 a 31.5) igual que la $\mathrm{HBr}$ (tres casos). Conclusiones: Indagar sobre los AMEG en un producto PEG parece ser útil en la inferencia de RCIU. Sin embargo, es insuficiente, por lo que en conjunto con otras herramientas nos ayuda a estimar posibles complicaciones y acciones preventivas.
\end{abstract}

Palabras clave: Bajo-peso al nacer. Restricción del crecimiento intrauterino. Pequeño para edad gestacional. Antecedentes maternos.

\section{Maternal prenatal history and neonatal risk complications for low-weight for gestacional age term newborns}

\section{Abstract}

Background: Low-birth-weight (LWB $<2.5 \mathrm{~kg}$ or $<10$ percentile) could be caused by constitutional matters or by intrauterine

\section{Correspondencia:}

*Mario Enrique Rendón-Macías

E-mail: drmariorendon@gmail.com
Disponible en internet: 10-08-2018 Bol Med Hosp Infant Mex. 2018;75:237-243 www.bmhim.com

1665-1146/@ 2018. Hospital Infantil de México Federico Gómez, impreso por Permanyer México SA de CV, todos los derechos reservados. 
growth restriction (IUGR), both with different neonatal complications. Without an adequate prenatal evaluation is hard to stablish those conditions. Knowing the maternal history for gestational diseases (MHGD) such as hypertension (HTA), hypothyroidism or diabetes, among others could help clarify that difference. The aim of this work was to determine if having a MHGD is associated to neonatal complications in newborns with LWB. Methods: Retrospective cohort study, which included 349 with LWB at term ( $\geq 37$ weeks of gestation [WG]) grouped into those with or without MHGD at a hospital nursery. The frequency of respiratory distress, hypoglycemia, and hyperbilirubinemia was determined. Results: 16.6\% (58/349) had MHGD (58.6\% for HTA and $41.3 \%$ hypothyroidism alone or combined). The neonate with MHGD were more borderline term (37 WGA, 55.2\% vs. $35.1 \%$; $p=0.037$ ), and had lower weight (difference of $\approx 100 \mathrm{~g} ; p=0.028$ ), had more cases with hypoglycemia (13.6\%; Cl 95\%: -4.08 to $31.2 \%$ ) but developed less respiratory distress (RD) (difference of $-4.7 \%$; $C / 95 \%$ : -20.6 to $11.05 \%$ ). Hypoglycemia in 39 WGA was only seen among neonates without MHGD (difference 12.7\%; C/95\%:3.9 to 31.5\%) just as for the hyperbilirubinemia cases (three).Conclusions: Inquiring about the MHGD on LBW term babies could be useful in the inference of IUGR, although we need other tools so that altogether can help to predict possible complications and to plan preventive actions.

Key words: Low-birth weight. Intrauterine growth restriction. Maternal history of gestational disease.

\section{Introducción}

La Organización Mundial de la Salud ha recalcado la importancia del neonato de bajo peso al nacer (NBPN) $(<2.5 \mathrm{~kg})$, debido al riesgo 20 veces mayor de morir en la etapa neonatal comparado con neonatos de peso adecuado ${ }^{1-3}$. Sin embargo, por la influencia de la edad gestacional sobre el peso, actualmente se define como tal cuando el peso se encuentra por debajo de dos desviaciones estándar de la media o debajo del percentil 10 para la edad gestacional ${ }^{2,4-6}$. Sin embargo, este riesgo puede diferir por la causa de la deficiencia ponderal. De acuerdo con lo anterior, se clasifica en pequeño para la edad gestacional (PEG), generalmente por causas constitucionales como sexo o etnia ${ }^{7}$ y cuando por evaluación ecográfica durante su etapa fetal mantuvo sus biométricos siempre por debajo de este percentil pero conservando un crecimiento constante hasta su nacimiento; y en recién nacidos con restricción en el crecimiento intrauterino (RCIU), cuando se demostró un crecimiento fetal por debajo de su potencial genético por debajo del percentil 3 y acompañado de alteraciones en el flujo placentario, uterino o fetal por ultrasonido Doppler, secundario a una causa placentaria, materna 0 alguna malformación fetal ${ }^{1,4,6}$. A su vez, la RCIU puede presentarse de forma simétrica (la cabeza y el abdomen son disminuidos proporcionalmente por hipoplasia celular en los órganos) 0 asimétrica (la cabeza es mayor al abdomen por redistribución del gasto cardíaco hacia órganos vitales durante una hipoxia crónica) $)^{1}$. Esto último explica el $80 \%$ de los casos. Así mismo, en los neonatos con RCIU se ha encontrado una mayor frecuencia de complicaciones posnatales, como hipoglucemia $(\mathrm{HG})$, hiperbilirrubinemia $(\mathrm{HBr})$, policitemia, dificultad respiratoria (DF), entre otras ${ }^{1,3,5}$.
Por otro lado, en muchas ocasiones es difícil establecer cuando el bajo peso de un neonato se debió a RCIU si no hubo un seguimiento ultrasonográfico fetal-placentario prenatal adecuado, salvo en los pacientes con malformaciones evidentes.

Estudios previos han mostrado que diversas condiciones maternas, como la edad de la madre ( $<16$ años $0>35$ años), un bajo nivel socioeconómico, un índice de masa corporal pregestacional bajo, la presencia de anemia, enfermedad hipertensiva del embarazo, diabetes mellitus, hipotiroidismo o adicciones, como alcoholismo o tabaquismo, se han asociado con PEG, y sobre todo a $\mathrm{RCIU}^{1,2,8,9}$.

En general, no es raro que los neonatólogos y pediatras se enfrenten a la atención de un neonato PEG sin disponer de información sobre el crecimiento intrauterino, y aunque pueden sospechar la causa del bajo peso (RCIU), sobre todo si hay un crecimiento asimétrico, en muchas ocasiones tal precisión es falible. Dada la alta frecuencia de antecedentes maternos en los neonatos con RCIU con mayor riesgo posnatal, estos podrían utilizarse como marcadores para una vigilancia más estrecha o de acciones preventivas.

Basándose en lo anterior, en este estudio se determinó si la existencia de antecedentes maternos gestacionales en niños con bajo peso al nacer se asocia con una mayor tasa de complicaciones posnatales tempranas. De ser así, su indagación y confirmación impactará en acciones preventivas primarias o secundarias otorgadas por los médicos que atiendan a estos neonatos.

\section{Métodos}

Se realizó un estudio de cohorte retrospectivo. Se incluyeron 349 recién nacidos vivos de término ( $\geq 37$ 
semanas de edad gestacional [SEG] obtenido por fecha de última menstruación) y con un peso por debajo del percentil 10 según las tablas de Jurado ${ }^{10}$, que son utilizadas para clasificar a los recién nacidos en esta unidad. Para calcular el tamaño de la muestra, se asumió un mínimo de $15 \%$ de madres con al menos un antecedente prenatal, con una incidencia esperada de HG (complicación más probable) en estos neonatos de, al menos, el $30 \%$ y con una diferencia esperada de un riesgo de 2.3, un poder estadístico del $80 \%$ y un riesgo de error tipo I de $p<0.05$. Se estimó un mínimo de 58 neonatos hijos de madres con algún antecedente y 250 sin antecedentes (www. sample-size.net). Se excluyeron productos de embarazos múltiples, prematuros en estado crítico (ingresados a la terapia intensiva neonatal), con malformaciones congénitas o cromosomopatías y aquellos programados a un procedimiento quirúrgico en el posnatal inmediato $(<48 \mathrm{~h})$. De todos los neonatos se registró el sexo, la edad gestacional, la vía de nacimiento, el Apgar a los cinco minutos y el peso y talla al nacer.

Como parte de la vigilancia estrecha de estos neonatos de bajo peso, todos fueron admitidos al cunero fisiológico y evaluados cada hora para determinar la presencia de alguno de los siguientes padecimientos: DR por cualquier causa, definida como el aumento de la frecuencia respiratoria por arriba de 60 respiraciones por minuto y algún dato de DR por la escala de Silverman Anderson ${ }^{11} ; \mathrm{HG}$, determinada por glucometría capilar con valores $<45 \mathrm{mg} / \mathrm{dl}$, con o sin datos clínicos (diaforesis, temblores o crisis convulsiva) ${ }^{12}$. En todos los pacientes, la medición se realizó al nacimiento, a los 30 y 60 minutos después, o en cualquier momento si hubo síntomas; $\mathrm{HBr}$, determinada solamente en aquellos pacientes con ictericia (Kramer II) $)^{13,14}$ y confirmada por la elevación de la bilirrubina indirecta sérica por arriba de los valores críticos según la norma ${ }^{13,14}$. Esta vigilancia se mantuvo hasta el alta o si estas ameritaron el internamiento en la Unidad de Cuidados Intensivos Neonatales (UCIN). Las complicaciones posteriores no fueron evaluadas.

Así mismo, de los expedientes de las madres se colectó la información sobre la edad, toxicomanías (tabaquismo, alcoholismo) y la presencia de cualquiera de los siguientes antecedentes prenatales: enfermedad hipertensiva del embarazo, diabetes gestacional, hipotiroidismo u otra, estableciendo el diagnóstico al cumplir los criterios establecidos en las Guías de Práctica Clínica.

\section{Análisis estadístico}

Los pacientes fueron agrupados basándose en la presencia de madres con antecedentes de enfermedades prenatales.

Todas las variables cualitativas fueron resumidas en frecuencias simples y relativas en porcentaje por grupo y contrastadas con prueba exacta de Fisher de dos colas $0 \chi^{2}$ en sus opciones según el tipo de variable (razón máxima verosimilitud o lineal por lineal). Para las variables cuantitativas, se resumieron en medianas con su rango (mínimo y máximo) o con medias y desviaciones estándar según su distribución de datos. La contrastación se realizó con la prueba $U$ de Mann Whitney o la t de Student no pareada, de acuerdo con los criterios anteriores.

Las complicaciones se calcularon como tasas de incidencia acumuladas al egreso o ingreso a UCIN en porcentajes para cada complicación (DR, $\mathrm{HG} \circ \mathrm{HBr}$ ) y establecidas por edades gestacionales $(37,38,39$ y 40-41 SEG).

Para determinar el efecto del antecedente prenatal de las madres en la mayor o menor presentación de las complicaciones, se calcularon los riesgos absolutos o diferencias de riesgos con sus intervalos de confianza al 95\% (IC 95\%). Se consideró una diferencia estadísticamente significativa ante la ausencia del valor de "0" dentro del intervalo.

Los análisis se realizaron con el paquete estadístico SPSS versión 20. El nivel de significación estadística fue establecido con una $p<0.05$.

\section{Resultados}

Cumplieron los criterios de inclusión 349 neonatos con bajo peso al nacer; de ellos, el 16.6\% (58/349) fueron hijos de madres con alguna enfermedad prenatal.

En la tabla 1 se observa la comparación del estado de las madres. Aunque no se alcanzó una significación estadística, las madres con algún antecedente fueron un año mayores $(p=0.06)$ y el $18 \%$ fueron mayores de 34 años $(p=0.012)$. Con respecto a la proporción de madres consumidoras tanto de alcohol, tabaco o ambos, no se encontraron diferencias estadísticamente significativas.

En el grupo de madres con enfermedades prenatales, la enfermedad hipertensiva sola o combinada, se presentó en más de la mitad. La segunda enfermedad más común consistió en el hipotiroidismo ( $\approx 40 \%$ ). La diabetes gestacional se presentó en el $8.5 \%$ de este grupo. 
Tabla 1. Características de las madres según los antecedentes de enfermedad prenatal

\begin{tabular}{|c|c|c|c|c|}
\hline & $\begin{array}{c}\text { Con antecedentes } \\
(n=58)\end{array}$ & $\begin{array}{c}\text { Sin antecedentes } \\
(\mathbf{n}=291)\end{array}$ & $\begin{array}{c}\text { Total } \\
(n=349)\end{array}$ & Valor de $p$ \\
\hline $\begin{array}{l}\text { Edad (años) } \\
\text { Mediana (min-máx) } \\
\text { Madres > } 34 \text { años }\end{array}$ & $\begin{array}{l}35(16-44) \\
58.6 \%(34)\end{array}$ & $\begin{array}{c}34(18-54) \\
40.5 \%(118)\end{array}$ & $\begin{array}{c}34(16-54) \\
43.6 \%(152)\end{array}$ & $\begin{array}{l}0.06^{*} \\
0.012^{\dagger}\end{array}$ \\
\hline $\begin{array}{l}\text { Toxicomanías } \\
\text { Tabaquismo } \\
\text { Alcoholismo } \\
\text { Tabaquismo y alcoholismo }\end{array}$ & $\begin{array}{c}10.3 \%(6) \\
0 \\
1.7 \%(1)\end{array}$ & $\begin{array}{c}9.7 \%(27) \\
0.3 \%(1) \\
5.8 \%(17)\end{array}$ & $\begin{array}{c}9.5 \%(33) \\
0.3 \%(1) \\
5.2 \%(18)\end{array}$ & $0.24^{\ddagger}$ \\
\hline $\begin{array}{l}\text { Enfermedad demostrada } \\
\text { Enfermedad hipertensiva } \\
\text { Hipotiroidismo } \\
\text { Diabetes gestacional } \\
\text { Hipotiroidismo y diabetes } \\
\text { Hipertensión e hipotiroidismo } \\
\text { Hipertensión y diabetes } \\
\text { Anemia de células falciformes } \\
\text { HTA + DG + hipotiroidismo }\end{array}$ & $\begin{array}{c}51.7 \%(30) \\
32.8 \%(19) \\
3.4 \%(2) \\
3.4 \%(2) \\
3.4 \%(2) \\
1.7 \%(1) \\
1.7 \%(1) \\
1.7 \%(1)\end{array}$ & $\begin{array}{l}- \\
- \\
- \\
- \\
- \\
-\end{array}$ & & \\
\hline
\end{tabular}

En cuanto a las características de los recién nacidos, en ambos grupos predominó el sexo femenino (64.2\%). A pesar de que todos los neonatos fueron de término, los hijos de madres con antecedentes fueron, en su mayoría (55.2\%), de 37 SEG, y 38 SEG para los hijos de madres sin enfermedad prenatal $(p=0.037)$. Con respecto a la vía de nacimiento, no hubo diferencias entre los grupos, y en ambos predominó la resolución por cesárea. Ningún paciente tuvo un Apgar $<7$, y el $91.1 \%$ fue de 9 a los cinco minutos.

Con respecto a la somatometría al nacimiento, en la evaluación del peso, con excepción de los neonatos de 37 SEG, no hubo diferencias estadísticamente significativas entre ambos grupos. En los neonatos de 37 SEG, el peso de los hijos de madres con enfermedad prenatal fue menor con respecto a las sanas (diferencia de $\approx 100 \mathrm{~g} ; \mathrm{p}=0.028$ ). Por otro lado, la talla también fue muy semejante entre los grupos, con excepción de Ios neonatos de 39 SEG; en ellos, nuevamente, la talla fue menor en los hijos de madres con enfermedad prenatal (diferencia $\approx 1 \mathrm{~cm} ; \mathrm{p}=0.039$ ) (Tabla 2).

\section{Complicaciones posnatales}

Para la DR se encontró una menor incidencia en los neonatos hijos de madres con enfermedades en los recién nacidos de 37 y 38 SEG. En particular, no hubo casos en los neonatos de 38 SEG hijos de madres con alguna enfermedad (< 10\% casos; IC 95\%: 5 a 16). Para los neonatos de 39 o más SEG, la frecuencia fue semejante entre los grupos, con un porcentaje poco mayor si había el antecedente materno (Tabla 3).

En cuanto a la presentación de $H G$, todas fueron detectadas por medición capilar dentro de las primeras seis horas de vida y sin diferencia entre grupos (datos no mostrados) y ningún paciente tuvo datos clínicos asociados. En los pacientes con antecedente en sus madres, solo se presentó en los de 37 y 38 SEG y en mayor proporción a los hijos de madres sanas, aunque las diferencias no alcanzaron la significación estadística. A partir de las 39 SEG, solo se vieron casos en el grupo de madres sanas $(p<0.05)$.

La presencia de $\mathrm{HBr}$ se encontró solo en tres pacientes del grupo de madres sanas. Se presentó en neonatos de 37 y 38 SEG. En todos ellos fue necesario el manejo con fototerapia por 24 horas y fueron egresados sin complicaciones.

\section{Discusión}

EI NBPN sigue siendo un reto para el manejo tanto del pediatra como del neonatólogo, más aún si no se puede establecer prenatalmente si esta merma ponderal es por factores propios del producto o por una agresión de su entorno. Los presentes datos apoyan que el conocer los antecedentes maternos puede ayudar a 
Tabla 2. Características de los neonatos de bajo peso para edad gestacional según los antecedentes de enfermedad prenatal

\begin{tabular}{|c|c|c|c|c|}
\hline & $\begin{array}{l}\text { Con antecedente materno } \\
\qquad(\mathrm{n}=\mathbf{5 8})\end{array}$ & $\begin{array}{l}\text { Sin antecedente materno } \\
\qquad(\mathrm{n}=291)\end{array}$ & $\begin{array}{c}\text { Total } \\
(\mathrm{n}=349)\end{array}$ & Valor de $p$ \\
\hline $\begin{array}{l}\text { Sexo } \\
\text { Femenino } \\
\text { Masculino }\end{array}$ & $\begin{array}{l}63.8 \%(37) \\
36.2 \%(21)\end{array}$ & $\begin{array}{l}64.3 \%(187) \\
35.7 \%(104)\end{array}$ & $\begin{array}{l}64.2 \%(224) \\
35.8 \%(125)\end{array}$ & $1.00^{*}$ \\
\hline $\begin{array}{l}\text { Semanas de gestación (SDG) } \\
37 \\
38 \\
39 \\
40 \\
41\end{array}$ & $\begin{array}{c}55.2 \%(32) \\
25.9 \%(15) \\
12.1 \%(7) \\
6.9 \%(4) \\
0\end{array}$ & $\begin{array}{c}35.1 \%(102) \\
39.2 \%(114) \\
18.9 \%(55) \\
5.2 \%(15) \\
1.7 \%(5)\end{array}$ & $\begin{array}{c}38.4 \%(134) \\
37.0 \%(129) \\
17.8 \%(62) \\
5.4 \%(19) \\
1.4 \%(5)\end{array}$ & $0.037^{\dagger}$ \\
\hline $\begin{array}{l}\text { Resolución del embarazo } \\
\text { Cesárea } \\
\text { Parto }\end{array}$ & $\begin{array}{l}82.8 \%(48) \\
17.2 \%(10)\end{array}$ & $\begin{array}{l}79.7 \%(232) \\
20.3 \%(59)\end{array}$ & $\begin{array}{c}80.2 \%(280) \\
19.8 \%(69)\end{array}$ & $0.59^{*}$ \\
\hline $\begin{array}{l}\text { Peso al nacimiento }(g) \\
37 \text { SDG } \\
38 \text { SDG } \\
39 \text { SDG } \\
40 \text { SDG }\end{array}$ & $\begin{array}{c}2284 \pm 222 \\
2434 \pm 132 \\
2496 \pm 124 \\
2607 \pm 75\end{array}$ & $\begin{array}{c}2380 \pm 148 \\
2488 \pm 174 \\
2528 \pm 113 \\
2611 \pm 71\end{array}$ & & $\begin{array}{c}0.028^{\ddagger} \\
0.169^{\ddagger} \\
0.54^{\ddagger} \\
0.092^{\ddagger}\end{array}$ \\
\hline $\begin{array}{l}\text { Talla al nacimiento }(\mathrm{cm}) \\
37 \text { SGD } \\
38 \text { SDG } \\
39 \text { SDG } \\
40 \text { SDG }\end{array}$ & $\begin{array}{l}46.1 \pm 1.5 \\
46.8 \pm 1.3 \\
46.8 \pm 1.4 \\
47.2 \pm 0.9\end{array}$ & $\begin{array}{l}46.5 \pm 1.4 \\
47.1 \pm 1.4 \\
47.9 \pm 1.2 \\
47.6 \pm 1.2\end{array}$ & & $\begin{array}{l}0.225^{\ddagger} \\
0.464^{\ddagger} \\
0.039^{\ddagger} \\
0.542^{\ddagger}\end{array}$ \\
\hline $\begin{array}{l}\text { APGAR (a los } 5 \text { minutos) } \\
7 \\
8 \\
9 \\
10\end{array}$ & $\begin{array}{c}0 \\
3.4 \%(2) \\
91.4 \%(53) \\
5.2 \%(3)\end{array}$ & $\begin{array}{c}0.3 \%(1) \\
4.5 \%(13) \\
91.1 \%(265) \\
5.2 \%(3)\end{array}$ & $\begin{array}{c}0.3 \%(1) \\
4.3 \%(15) \\
91.1 \%(318) \\
4.3 \%(15)\end{array}$ & $0.54^{\dagger}$ \\
\hline
\end{tabular}

*Prueba exacta de Fisher dos colas.

${ }^{\dagger}$ Prueba $\mathrm{X}^{2}$ lineal por lineal.

${ }^{\ddagger}$ Media \pm DS, T de Student.

Tabla 3. Complicaciones posnatales de los neonatos de bajo peso para edad gestacional según los antecedentes de enfermedad prenatal

\begin{tabular}{|c|c|c|c|}
\hline & Con antecedente materno & Sin antecedente materno & Diferencia (IC 95\%) \\
\hline $\begin{array}{l}\text { Síndrome de dificultad respiratoria } \\
37 \text { SDG } \\
38 \text { SDG } \\
39 \text { SDG } \\
40 \text { SDG }\end{array}$ & $\begin{array}{c}18.8 \%(6 / 32) \\
0 \%(0 / 15) \\
28.6 \%(2 / 7) \\
25.0 \%(1 / 4)\end{array}$ & $\begin{array}{c}23.5 \%(24 / 102) \\
10.5 \%(12 / 114) \\
10.9 \%(6 / 55) \\
20.0 \%(3 / 15)\end{array}$ & $\begin{array}{c}-4.7 \%(-20.6 \text { a } 11.05 \%) \\
-10.5 \%(-4.8 \text { a }-16.1 \%) \\
17.8 \%(-16.8 \text { a } 52.1 \%) \\
5 \%(-42 \text { a } 52 \%)\end{array}$ \\
\hline $\begin{array}{l}\text { Hipoglucemia } \\
37 \text { SDG } \\
38 \text { SDG } \\
39 \text { SDG } \\
40 \text { SDG }\end{array}$ & $\begin{array}{c}31.3 \%(10 / 32) \\
26.7 \%(4 / 15) \\
0 \%(0 / 7) \\
0 \%(0 / 4)\end{array}$ & $\begin{array}{c}17.6 \%(18 / 102) \\
14.0 \%(16 / 114) \\
12.7 \%(7 / 55) \\
0 \%(0 / 15)\end{array}$ & $\begin{array}{c}13.6 \%(-4.08 \text { a } 31.2 \%) \\
12.6 \%(-10.6 \text { a } 35.9 \%) \\
-12.7 \%(-3.9 \text { a }-21.5 \%) \\
-\end{array}$ \\
\hline $\begin{array}{l}\text { Hiperbilirrubinemia } \\
37 \text { SDG } \\
38 \text { SDG } \\
39 \text { SDG } \\
40 \text { SDG }\end{array}$ & $\begin{array}{l}0 \%(0 / 32) \\
0 \%(0 / 15) \\
0 \%(0 / 7) \\
0 \%(0 / 4)\end{array}$ & $\begin{array}{c}1.0 \%(1 / 102) \\
1.8 \%(2 / 114) \\
0 \%(0 / 55) \\
0 \%(0 / 15)\end{array}$ & $\begin{array}{c}-0.9 \%(-2.8 \text { a } 0.9 \%) \\
-1.7 \%(-4.1 \text { a } 0.6 \%) \\
-\end{array}$ \\
\hline
\end{tabular}

IC 95\% = Intervalo de confianza al 95\%; SDG: semanas de gestación. 
predecir sus posibles complicaciones posnatales tempranas. En primer lugar, los NBPN de madres "enfermas" fueron, en su mayoría, obtenidos por cesáreas realizadas más tempranamente (> 50\% a las $37 \mathrm{SEG}$ ). Así mismo, comparados con sus contrapartes de menores edades gestacionales ( 37 y $38 \mathrm{SEG}$ ) pesaron menos al nacer (37 SEG, $p<0.02$; y $38 \mathrm{SEG}, p=0.16$ ), aun con tallas semejantes, y exhibieron un mayor riesgo de HG. Sin embargo, aparentemente alcanzaron un nivel de maduración pulmonar mayor manifestado por una menor proporción de DR. Por otro lado, los neonatos más maduros (39 a 41 SEG), hijos de madres con antecedentes prenatales, fueron de menor talla. Este grupo, además, no presentó ningún evento de $H G$ neonatal.

La presencia de DR fue alta en ambos grupos, sobre todo en los de 37 y $38 \mathrm{SEG}$. Finalmente, la $\mathrm{HBr}$ no se asoció con el antecedente y fue una complicación rara en los NBPN que se estudiaron.

Como ha sido ya referido en la literatura, la edad materna ha sido ampliamente relacionada con el riesgo de bajo peso neonatal ${ }^{1,4}$. Por ello, no fue de extrañar que la mediana de edad en las madres fue de 34.5 años, pero sí el encontrar una edad poco mayor (un año) en aquellas con enfermedades durante su gestación. Así mismo, la edad mayor a 34 años también se ha relacionado con un riesgo incrementado para padecer enfermedades gestacionales. En particular, la enfermedad hipertensiva del embarazo y el hipotiroidismo $0^{9,15-17}$, ambas enfermedades muy prevalentes en este estudio.

Tanto la enfermedad hipertensiva del embarazo como el hipotiroidismo suelen afectar el crecimiento fetal al alterar el flujo placentario y las hormonas necesarias para el desarrollo del producto, a diferencia de la diabetes mellitus, cuyo efecto principal es la macrosomía fetal $9,15,16,18$. La menor edad gestacional encontrada en los productos de estas madres se puede relacionar a la necesidad de interrumpir más temprano el embarazo ante el riesgo de morbilidad o mortalidad tanto de la madre como del producto ${ }^{16}$. Sin embargo, el observar un menor peso de sus hijos en comparación con sus contrapartes de igual edad gestacional, reafirma el impacto de estas enfermedades en el crecimiento fetal.

Con respecto a la vía del nacimiento, como se observó en estos pacientes, la obtención a través de cesárea fue altamente frecuente. En general, la decisión suele basarse en el alto riesgo de hipoxia neonatal por baja reserva fetal esperada en todo feto que no ha alcanzado un peso normal para la edad gestacional, sin considerar la recomendación obstétrica de este procedimiento en madres mayores de 35 años, sobre todo primigestas (condición frecuente en este estudio), 4,5,16. No se encontraron diferencias entre los grupos. Lo más trascendente fue la muy baja tasa de pacientes con Apgar menor a 7 a los 5 minutos de vida (solo un paciente): al no presentar sufrimiento fetal agudo o crónico, no se desarrolló un estado de hipoxia en el feto que derivara en policitemia con riesgo elevado de presentar $\mathrm{HBr}^{1,5,14}$. Por otro lado, es sabido que un nacimiento por vía cesárea en niños de término se asocia con síndrome de dificultad respiratoria (SDR) tipo $\|^{1,8}$. Esto pudo observarse claramente por una frecuencia del 20 y $25 \%$ de los pacientes hijos de madres con y sin antecedentes, respectivamente. Sin embargo, se pudo notar una mayor diferencia en los neonatos con menor edad gestacional (37 y 38 SEG). En ellos, la DR se presentó menos cuando había el antecedente de enfermedad materna. Algunos estudios han mostrado que el feto, al estar sometido a un estrés constante en un ambiente de hipoxia y sufrimiento fetal, presenta una maduración pulmonar más rápida, condición que los protege para desarrollar un síndrome de DR al nacer, aunque también incrementa el riesgo de síndrome de aspiración de meconio ${ }^{1,8}$.

Con respecto al riesgo de $\mathrm{HG}$ neonatal, es sabido que los NBPN cursan con bajas concentraciones de insulina intraútero, y por tanto, disminución en la síntesis y depósito de glucógeno hepático; por ello, al nacer, requieren de una alimentación de inicio temprana y más temprana entre mayor sea la severidad de la $\mathrm{RCIU}^{18}$. En este estudio se pudo observar una frecuencia un poco mayor de $H G$ en los recién nacidos de 37 a 38 SEG, sobre todo en aquellos con antecedentes maternos, aunque la diferencia no fue estadísticamente significativa. En contraparte, para los neonatos de 39 SEG, este evento fue muy raro y solo se presentó en el grupo de neonatos sin enfermedad materna. Es posible que los neonatos de 39 a 40 SEG hayan logrado abastecer sus reservas durante esas dos semanas extra de gestación.

Este estudio presentó varias fortalezas. Por un lado, la muestra resultó lo suficientemente amplia para contrastar el efecto de los antecedentes maternos más comunes sobre las complicaciones más usuales en los neonatos a término. Se siguió a estos pacientes durante toda su evolución en cuna, lo que permitió una vigilancia estrecha, además de intervenciones oportunas para evitar complicaciones mayores. En contraste, varias limitaciones se deben considerar en este trabajo. No se logró establecer una diferencia estricta entre los recién nacidos PEG y los recién nacidos con diagnóstico claro de restricción intrauterina. Lo anterior es importante debido a que permite esclarecer mejor los riesgos de complicaciones específicas. 
A pesar de estas limitaciones, los presentes datos apoyan la utilidad de evaluar los antecedentes maternos en todo producto de bajo peso. Como se observó en los resultados, cuando estos se presentan, es posible pensar en un producto que además de bajo peso pudiera tener una restricción al crecimiento, y por ende, mayor riesgo de presentar HG temprana. De no existir este antecedente, el neonato pudiera tener un crecimiento bajo por un componente constitucional, y el obtenerlo en un periodo de término muy limítrofe (37-38 SEG), lo pone en un riesgo de DR. Quizá en estos productos la conducta de continuar el embarazo sería una opción a pesar de estimarse un peso bajo. Por último, el manejo obstétrico y neonatal en todo neonato de bajo peso debe ser valorado en conjunto, y de preferencia apoyado en estudios de imagen prenatal que permitan una mejor decisión.

El indagar sobre los antecedentes maternos relacionados con enfermedades gestacionales, como la enfermedad hipertensiva del embarazo, el hipotiroidismo materno, entre otros, en una gestación con producto de bajo peso al nacer parece ser útil en la inferencia de la posible RCIU. Sin embargo, de manera aislada, no es un indicador confiable sobre el riesgo de complicaciones posnatales, por lo que la historia clínica prenatal, el control prenatal adecuado y la vigilancia estrecha de los recién nacidos en conjunto ayudarán a estimar posibles complicaciones y acciones preventivas.

\section{Responsabilidades éticas}

Protección de personas y animales. Los autores declaran que para esta investigación no se han realizado experimentos en seres humanos ni en animales.

Confidencialidad de los datos. Los autores declaran que han seguido los protocolos de su centro de trabajo sobre la publicación de datos de pacientes.

Derecho a la privacidad y consentimiento informado. Los autores declaran que en este artículo no aparecen datos de pacientes.

\section{Conflicto de intereses}

Los autores declaran no tener ningún conflicto de intereses.

\section{Bibliografía}

1. Arango F, Grajales J. Restricción en el crecimiento intrauterino. CCAP 2010;9:5-14.

2. Boguszewski M, Mericq V, Bergada I, Damiani D, Belgorosky A. Consenso Latinoamericano: niños pequeños para la edad gestacional. Rev Chil Pediatr. 2012;83:620-634.

3. Labarta J, de Arriba A. Consecuencias de nacer pequeño para la edad gestacional. Bol S Vasco-Nav Pediatr. 2013;45:32-33.

4. Pimiento L, Beltrán M. Restricción del crecimiento intrauterino: Una aproximación al diagnóstico, seguimiento y manejo. Rev Chil Obstet Ginecol. 2015;80:493-502

5. Gormaz M. Pequeño para la edad gestacional en el periodo neonatal. Rev Esp Endocrinol Pediatr. 2012;3:90-92.

6. Gómez M, Danglot $\mathrm{C}$, Aceves M. Clasificación de los niños recién nacidos. Rev Mex Pediatr. 2012;79:32-39.

7. Parca M, Peloso T, Dos Anjos M. Frequency and risk factors for the birth of small-for-gestacional-age newborns in a public maternity hospital. Einstein. 2016;14:317-323.

8. Castro F, Labarrere Y, González G, Barrios Y. Factores de riesgo del síndrome de dificultad respiratoria de origen pulmonar en el recién nacido. Rev Cubana Enfermer. 2007;23:1-15.

9. Salazar L, Gómez T, Bequer L, Heredia D. El bajo peso como consecuencia de la hipertensión inducida por el embarazo. Factores de riesgo. Rev Hosp Mat Inf Ramón Serdá. 2014;33:14-20.

10. Jurado E. El crecimiento intrauterino. Correlación peso/longitud corporal al nacimiento en función de la edad de gestación. Gac Med Mex. 1971;102:227-255.

11. Guía de Práctica Clínica. Diagnóstico y tratamiento de la taquipnea transitoria del recién nacido. Evidencias y recomendaciones. Catálogo maestro de guías de práctica clínica. IMSS 044-08. Disponible en: http:// www.cenetec.salud.gob.mx/descargas/gpc/CatalogoMaestro/044_GPC_ TaquipneaTransRN/IMSS_044_08_EyR.pdf

12. Guía de Práctica Clínica. Diagnóstico y tratamiento de la hipoglucemia neonatal transitoria. Evidencias y recomendaciones. Catálogo maestro de guías de práctica clínica. IMSS 442-11. Disponible en: http://www. cenetec.salud.gob.mx/descargas/gpc/CatalogoMaestro/446_GPC_Hipoglucemia_neonatal/GER_Hipoglucemia_neonatal.pdf

13. Guía de Práctica Clínica. Detección oportuna, diagnóstico y tratamiento de la hiperbilirrubinemia en niños mayores de 35 semanas de gestación hasta las 2 semanas de vida extrauterina. Evidencias y recomendaciones. IMSS 262-10. Disponible en: http://www.cenetec.salud.gob.mx/descargas/gpc/CatalogoMaestro/262_IMSS_10_Hiperbilirrubinemia/EyR_ IMSS_262_10.pdf

14. Mazzi E. Hiperbilirrubinemia neonatal, actualización. Rev Soc Bol Pediatr. 2005;44:26-35.

15. Ulanowick M, Parra K, Rosas G, Hipertensión Gestacional. Consideraciones generales, efectos sobre la madre y el producto de la concepción. Rev Posgr VI Cátedra Med. 2005;152:19-22.

16. Gómez M, Danglot C. El neonato de madre con preeclampsia-eclampsia. Rev Mex Pediatr. 2006;73:82-88.

17. Serrano M. Alteraciones de tiroides y embarazo: resultados perinatales. Rev Esp Med Quir. 2013;18:200-205.

18. Arizmendi J, Carmona V, Colmenares A, Gómez D, Palomo T. Diabetes gestacional y complicaciones neonatales. Rev Fac Med. 2012;20:50-60. 